\title{
El aprendizaje servicio como pedagogia inclusiva en estudiantes con necesidades educativas especiales
}

\author{
Italo Fiorin \\ Universitá LUMSA di Roma, Italia
}

\section{Carina Rossa}

Fondazione Pontificia Scholas Occurrentes. Universitá LUMSA di Roma, Italia.

\section{Resumen}

El paso del modelo médico al modelo social de los diferentes tipos de discapacidad comporta un cambio de paradigma gracias al cual el límite se entiende como un estado social y no como una condición medica. La escuela es uno de los principales y más importantes ámbitos de inclusión que incide de manera especial en el desarrollo personal. La inclusión escolar de los estudiantes con Necesidades Educativas Especiales (NEE) exige estrategias pedagógicas adecuadas y personalizadas. Al mismo tiempo se necesitan dinámicas que animen la participación de todos los estudiantes en proyectos comunes, en el pleno respeto de la diversidad, sin dejar atrás a ninguno y que favorezcan la relación entre todos los componentes de la clase y fuera de ella. En este trabajo de investigación se postula que el aprendizaje servicio es una propuesta educativa inclusiva para todos los estudiantes gracias a la cual también aquellos alumnos con NEE pueden alcanzar una plena inclusión como protagonistas de acciones de transformación social.

\section{Palabras clave}

Protagonismo estudiantil, prosocialidad, límite, transformación social. 


\title{
Service learning as inclusive pedagogy in students with special educational needs
}

\author{
Abstract \\ The change from the medical model to the social model of the different types of \\ disability entails a change of paradigm thanks to which the limit is understood as a \\ social state and not as a medical condition. The school is one of the main and most \\ important areas of inclusion that has a special impact on personal development. The \\ school inclusion of students with Special Educational Needs (SEN) requires \\ appropriate and personalized pedagogical strategies. At the same time, dynamics are \\ needed that encourage the participation of all students in common projects, in full \\ respect of diversity, without leaving any behind and that favor the relationship \\ between all the components of the class and beyond. In this research, it is postulated \\ that service learning is an inclusive educational proposal for all students, thanks to \\ which students with SEN can also achieve full inclusion as protagonists of social \\ transformation actions.
}

\section{Keywords}

Student protagonism, prosociality, limit, social transformation. 


\section{Introducción}

En las actividades con estudiantes que presentan algún tipo de desventaja, un obstáculo, a veces, puede estar representado por la visión que se tiene de ellos. De hecho, frecuentemente vienen considerados solo como destinatarios de nuestras atenciones y no como sujetos activos en los cuales hay que desarrollar su potencial. Es necesario encontrar una didáctica adecuada a través de la cual los estudiantes con necesidades educativas especiales (NEE) puedan convertirse en protagonistas de actividades de ayuda hacia los demás, siendo así actores primarios de transformación social y no solo receptores de ayuda. Una escuela no puede ser realmente acogedora e inclusiva hacia dentro si en la comunidad alrededor de ella permanecen obstáculos y barreras a causa de prejuicios y actitudes estigmatizantes que impiden la plena integración de niños y adolescentes desventajados. Además, es tarea de la escuela contribuir a la transformación de la sociedad con el objetivo que sea para todos.

En este sentido el aprendizaje servicio se presenta como una pedagogía inclusiva adecuada, que genera notables impactos en la calidad del aprendizaje, dando vida a profundos cambios en la comunidad que rodea la escuela, como también a los estudiantes que, por sus características de protagonismo y prosocialidad, emprenden un camino que los conduce hacia una participación activa y transformante.
La experiencia empírica y el corpus de investigación que muestran los efectos de la participación activa de los estudiantes con NEE en proyectos de aprendizaje servicio están en crecimiento sobre todo en países donde esta pedagogía está más difundida. Existen varios esquemas de participación que permiten identificar posibles modelos de aprendizaje servicio e inclusión ligados al contexto cultural. Sin embargo se advierte la necesidad de desarrollar un esquema de inclusión que involucre mayormente el territorio como lugar primario de inclusión. No se encuentra en la literatura una definición y descripción articulada de estos diferentes modelos, que podrían llevar a una aplicación más sistemática del aprendizaje servicio en el ámbito de la inclusión de personas con NEE.

\section{Hacia una definición de inclusión. Discusión entre los principales expertos}

La literatura científica ofrece diferentes enfoques del concepto de inclusión. La diversidad se asocia sobre todo con el contexto cultural del autor que describe los desafíos pedagógicos de su región y encuentra una coincidencia global en los principios expresados en los documentos internacionales.

El desafío de la inclusión es global. En los países con economías más pobres, la prioridad es el acceso a la educación en general, pero también a una educación de calidad; mientras que en los países más prósperos los desafíos se relacionan con la calidad de la educación, ya sea por la segregación de los estudiantes con NEE, como también

Fiorin, I.; Rossa, C. (2018). El aprendizaje servicio como pedagogia inclusiva en estudiantes con necesidades educativas especiales. RIDAS, Revista Iberoamericana de Aprendizaje Servicio, 5, 24-36. DOI10.1344/RIDAS2018.5.3 
por la recepción de múltiples formas del multiculturalismo presentes ahora en las escuelas.

En esta investigación se quiso comparar el pensamiento de diez expertos de inclusión educativa y social, elegidos entre quienes han elaborado los textos más conocidos en el campo científico y experimental, provenientes de las áreas geográficas que corresponden a las experiencias seleccionadas como muestra en el análisis en el que se enfoca este trabajo. Las naciones son los Estados Unidos, España, Italia, Chile y Argentina. Se añade, además, un autor inglés, Mel Ainscow, reconocido por su intervención en la extensión de documentos internacionales de la UNESCO que han incidido mucho en el concepto global de inclusión. Los autores, en orden alfabético, son Mel Ainscow y Tony Booth (Inglaterra); Rebeca Anijovich (Argentina); Rosa Blanco (Chile); Andrea Canevaro (Italia); Italo Fiorin (Italia); Carmen García Pasto y José Gimeno Sacristán (España); Darío Ianes (Italia); David Johnson y Roger Johnson (Estados Unidos) y Giuseppe Milan (Italia).

Para el análisis de los diferentes enfoques se utilizó un método de educación comparativa, basado en el modelo de referencia para la encuesta comparativa en tres dimensiones de Bob Adamson y Paul Morris (2009). Las tres dimensiones (metas y perspectivas, enfoque y contenido, manifestaciones y principios de enseñanza) están interconectadas. Se trata de comparar la finalidad, es decir la filosofía de base. El hecho de tener un propósito implica la adopción de una perspectiva, mientras que el propósito también inspira las preguntas a las cuales el autor espera responder; preguntas que, a su vez, sugieren un punto clave, el enfoque, los contenidos, los conceptos claves que se expresarán en las actividades de enseñanza.

Figura 1: Modelo para la encuesta comparativa tridimensional de Bob Adamson y Paul Morris

Finalidad y prospectivas

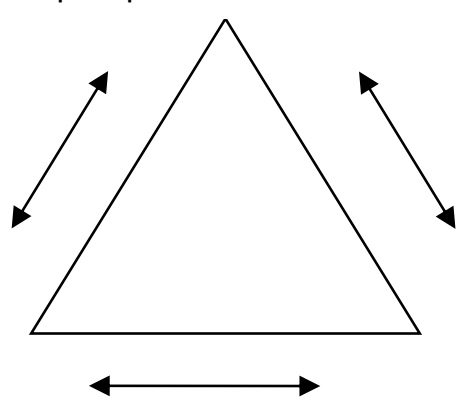

Focus y contenido

Manifestaciones y principios didácticos

\subsection{Enfoques sobre la inclusión}

La definición más frecuente de inclusión está inspirada en la idea de Ainscow vista como una forma de crecimiento ilimitado del aprendizaje y la participación de todos los estudiantes. Para Ainscow (2007) la inclusión es para todos los alumnos que, por alguna razón, encuentran obstáculos para el aprendizaje o la participación. Su promoción requiere un cambio en el contexto, en las culturas, en las políticas educativas y en las prácticas. La identificación y eliminación de las barreras dentro de los contextos sociales y educativos afectan los aspectos físicos de la arquitectura, pero también los mentales y culturales (prejuicios, hábitos, etc.). El éxito académico de todos los estudiantes

Fiorin, I.; Rossa, C. (2018). El aprendizaje servicio como pedagogia inclusiva en estudiantes con necesidades educativas especiales. RIDAS, Revista Iberoamericana de Aprendizaje Servicio, 5, 24-36. DOI10.1344/RIDAS2018.5.3 
(incluso aquellos con NEE) y la participación total serán indicadores de la verdadera inclusión. La investigación ha demostrado que la influencia de la familia y la escuela explica la calidad del aprendizaje y que las dificultades pueden tener otras causas además de la discapacidad. Si la escuela se reorganiza, los estudiantes podrían aprender de manera efectiva, incluso aquellos con NEE.

Ainscow (2007) sostiene además que una verdadera inclusión depende de la presencia, la participación y el éxito de todos los estudiantes. El término presencia tiene que ver con el lugar donde el niño se educa, la constancia y puntualidad en asistir a clase; la participación se refiere a la calidad de su experiencia, por lo tanto, a la voz de los estudiantes, mientras que el rendimiento escolar tiene que ver con los resultados del aprendizaje a lo largo del plan de estudios.

En este ámbito cabe destacar el importante aporte de Booth y Ainskow (2002), autores del Índice para la inclusión, el manual de guía traducida a varios idiomas que se ha convertido, en los últimos años en un punto de referencia a nivel internacional en cuanto al desarrollo del diseño inclusivo en las escuelas. En Italia, el índice fue editado por Fabio Dovigo y Dario Ianes (2008).

Para Ianes (2006), la inclusión implica las diferentes prácticas de respuesta personalizadas realizadas en las diversas necesidades educativas de todos los alumnos, incluso de aquellos con necesidades educativas especiales. La inclusión es, por lo tanto, más amplia que la integración. Evaluando, de hecho, todas las necesidades educativas especiales, viene la necesidad de responder de una manera inclusiva y el deseo de considerar las necesidades de todos los alumnos. Una respuesta verdaderamente inclusiva es una respuesta individualizada según sea necesaria. Decir que la inclusión también incluye integración significa reconocer que las condiciones del contexto, ofrecido por una escuela que sea realmente acogedora o inclusiva para todos los alumnos, son al mismo tiempo las condiciones ideales para la integración de los alumnos con discapacidad. La sola presencia de estos estudiantes ha llevado a la escuela a replantearse en términos de flexibilidad organizativa y didáctica, a cambiar para reflejar más las solicitudes nuevas y especiales, descubriendo así que, en realidad, todos los estudiantes obtienen beneficios de una enseñanza renovada y más atenta a las diferencias individuales.

Blanco (2007) comparte la definición de Ainscow pero enfatiza que la educación inclusiva es ante todo una cuestión de derecho. La inclusión significa ofrecer educación de calidad para todos aquellos que se encuentran en una situación de desventaja o vulnerabilidad. Hacer efectivo el derecho a la educación implica garantizar la igualdad de oportunidades, la participación y la no discriminación, y el derecho a la propia identidad. Participar significa aprender junto con otros y colaborar a través de experiencias de aprendizaje compartidas; implica tener voz y ser

Fiorin, I.; Rossa, C. (2018). El aprendizaje servicio como pedagogia inclusiva en estudiantes con necesidades educativas especiales. RIDAS, Revista Iberoamericana de Aprendizaje Servicio, 5, 24-36. DOI10.1344/RIDAS2018.5.3 
aceptado en la propia identidad, en libertad, autonomía y autogestión. Requiere participación activa en el aprendizaje y una conciencia de cómo se experimenta la educación. En un sentido más amplio y profundo, se trata de ser reconocido, aceptado y apreciado por uno mismo. La aplicación de esta ley, en la educación, es asegurar que todos los niños pueden ser educados juntos en la escuela, independientemente de las características sociales, culturales y personales. La escolarización en grupos especiales debería ser la excepción. Debe asegurarse, en la medida de lo posible, una enseñanza equivalente al plan de estudios común.

Gimeno Sacristán (1999) define la condición humana variada, porque somos diferentes los unos de los otros. Sin embargo, todos cambiamos constantemente, especialmente en las condiciones sociales y culturales posmodernistas, caracterizadas por la complejidad, la inestabilidad y la variedad de relaciones que establecemos en contextos cambiantes. A pesar de ello, la educación, que sigue siendo heredera de la iluminación, mantiene valores, habilidades y significados compartidos que respaldan una cierta homogeneidad en el pensamiento, en los valores y en comportamiento. Para incluir, para esperar la diversidad, uno debe tener el coraje de romper la forma predominante de organización escolar.

Para Fiorin (2016) inclusión es acogida. La escuela es inclusiva cuando no establece condiciones a priori, sino que acoge incondicionalmente cada alumno y lo pone en el centro de su atención.
Solo si una persona se siente bienvenida, así como es, puede sentirse segura, no amenazada por un ambiente hostil. La sensación de seguridad, de protección, le permite de manera gradual sentirse parte de la comunidad que lo ha recibido, por lo tanto, puede compartir sus valores y sentirse motivado para seguirlos, haciendo una síntesis de las expectativas personales o sociales. La necesidad de ser acogido, valorado, para alcanzar el éxito, se aplica a todos los estudiantes, cada uno involucrado en la empresa difícil del crecimiento, cada uno buscando realizarse a si mismo.

La elección de ser inclusivos requiere una mayor inversión; de generosidad y de compromiso para los docentes, pero también, y sobre todo, de recursos por parte de los responsables políticos (asegurando una mayor capacitación, condiciones organizativas sostenibles y asegurando recursos humanos y materiales...). La opción inclusiva es compatible con la escuela de calidad, pero no es gratuita.

La novedad que aporta Fiorin (2016) se refiere a la estrecha relación que existe entre la inclusión escolar y la inclusión social. La verdadera inclusión es el resultado de un pacto social, un esfuerzo conjunto entre la escuela y el territorio, preparadas para terminar con las barreras a la inclusión y promover la superación de la injusticia y de las sanciones sociales que provienen de su propia condición. En la escuela inclusiva los estudiantes se ponen al servicio del bien común.

Para Canevaro (2006) la inclusión es el encuentro con la diversidad, es la

Fiorin, I.; Rossa, C. (2018). El aprendizaje servicio como pedagogia inclusiva en estudiantes con necesidades educativas especiales. RIDAS, Revista Iberoamericana de Aprendizaje Servicio, 5, 24-36. DOI10.1344/RIDAS2018.5.3 
superación de las barreras que ponen obstáculos a las relaciones entre las personas, es búsqueda de reciprocidad. Los límites se ven como una posibilidad de relación; pueden indicar espacios vacíos, que resaltan los espacios plenos y que, a su vez, pueden llamar la atención. La exclusión se puede explicar como el completo vacío (el vacío social, el vacío dejado por los otros, el vacío de sentido), mientras que la inclusión es el pleno (plenitud social, plenitud de los otros, plenitud de significado). Para crecer y vivir necesitamos ambas realidades, en un contexto de reciprocidad necesaria. La dinámica del intercambio es de aceptación y rechazo debido a las diferencias possibles, cada uno reconoce en el otro algo de aceptable y algo que rechaza y, a su vez, es visto así por el otro. Esto se logra en un reconocimiento mutuo. La dinámica del intercambio es posible gracias a esta trama que no polariza el bien y el mal.

Para García Pastor (2003), la inclusión es acogida; significa entrar en el territorio del otro. La inclusión significa sentirse comunidad. Una escuelacomunidad se siente parte de una comunidad más grande, hacia la cual se abre y en la que se aprecia cada miembro, mediante la estimulación y el uso de los dones y talentos de cada uno, la promoción de la autoestima, el respeto y la interdependencia positiva. La inclusión es diálogo con el otro. Se trata de una receptividad del otro en mí. Un elemento distintivo que introduce el autor es el protagonismo. Necesitamos escuchar la voz de las personas con discapacidades; estar atento a lo que piensan, sus opiniones deben estar en el centro del debate sobre las personas con NEE.

En línea con la idea de inclusión entendida como relación autentica, Giuseppe Milan (2000) sostiene que la inclusión es una dimensión fundamental de la relación yo-tú, la dialógica. La relación yo-tú es propiamente inclusiva, mientras que en la relación no auténtica yo-esto actúan las trampas identitarias de la exclusión (la trampa individualista, la trampa de la asimilación, de la intolerancia, del asistencialismo, del sectarismo, etc.). La inclusión, en la relación educativa yo-tú, se lleva a cabo dentro de una relación dialógica asimétrica, que destaca una diferencia en los roles y debe moverse en una transición lenta, compleja e irresistible desde la asimetría a la simetría. Solo el educador puede primero comprender por completo a la otra parte, incluir al alumno. Sin embargo, el educador mismo, en virtud de su capacidad dialógica, pasa de extra a intra, convirtiéndose en un tú interno. El estudiante lo encuentra en sí mismo, incluye una comprensión siempre mayor hasta que la relación se acerque a la mutua inclusión. En este viaje de relación auténtica y creativa, entra en juego la fantasía real (buceo valiente en la orilla opuesta, frente a ella, pero sabiendo cómo viajar y comprender, haciendo presente al otro: empatia). $Y$ entra en juego, concretamente, lo que Buber (Milan, 2000) Ilama lucha, una lucha apasionada para ayudar al otro a emerger, a convertirse en lo que puede y debe ser, sin excluir a nadie. No sería inclusión si no fuera combate. La inclusión es la capacidad de ampliar la

Fiorin, I.; Rossa, C. (2018). El aprendizaje servicio como pedagogia inclusiva en estudiantes con necesidades educativas especiales. RIDAS, Revista Iberoamericana de Aprendizaje Servicio, 5, 24-36. DOI10.1344/RIDAS2018.5.3 
mirada, la mente, el corazón, nuestro territorio cultural para abrazar a aquellos que de lo contrario, debido a visiones sectarias y parciales, serían excluidos.

\subsection{Hacia una definición de inclusión}

Una definición de inclusión, inclusiva de los diversos enfoques, continúa siendo la expresión acuñada por el documento Educación para Todos de la UNESCO (2003). El todos es una expresión de la mas amplia diversidad, de la más profunda fragilidad, de los que están en desventajas por razones de injusticia y pobreza, de los que no tienen las mismas oportunidades de acceso a los bienes materiales y culturales. La inclusión significa eliminar obstáculos y barreras para que todos puedan aprender y participar. Inclusión es hospitalidad, es encuentro, es diálogo con el otro diferente de mí, es reciprocidad, donde cada uno incluye al otro en términos de dar y recibir. La inclusión es la libre participación en la comunidad social mediante la superación de obstáculos y barreras que impiden el crecimiento y desarrollo de los ciudadanos, donde todos actúan como responsables de su transformación social local y global.

\subsection{Definición de necesidad educativa especial}

Según Ianes (2006) una necesidad educativa especial es cualquier dificultad de desarrollo, en el contexto educativo y de aprendizaje, expresada en el funcionamiento también para el sujeto, en términos de daño, obstáculo o estigma social, independientemente de su etiología, y que requiere educación especial individualizada.

El área de NEE incluye tres

subcategorías principales: discapacidad (física, mental e intelectual); trastornos de aprendizaje específicos; y

desventaja social y cultural. En esta área incluimos, por lo tanto, todos los diversos trastornos/dificultades de aprendizaje, comportamiento y otros problemas relacionados con razones psicológicas o ambientales, de causas endógenas o exógenas que en conjunto comprometen el funcionamiento humano del estudiante.

Los sujetos con necesidades educativas especiales, por lo tanto, son todas aquellas personas en la edad de desarrollo en las que las necesidades educativas normales encuentran una mayor complejidad en la búsqueda de respuestas debido a alguna dificultad en su funcionamiento humano.

Si pudiéramos hacer una lista detallada de las diferentes NEE encontraríamos que esta lista no tiene fin. Para confirmar esto, es suficiente mencionar solo aquellos que pertenecen a la categoría de psicopatología del desarrollo. Estamos hablando de disturbios de la inteligencia; trastornos generalizados del desarrollo (trastorno del espectro autista, síndrome de Asperger); trastornos del comportamiento (agresivos hasta actos autolesivos, intimidación, trastornos del comportamiento alimentario, trastornos de conducta, oposición, delincuencia, consumo de drogas, etc.); trastorno por déficit de atención/hiperactividad; trastornos de ansiedad; fobias; trastorno obsesivo-compulsivo; mutismo selectivo; trastornos del

Fiorin, I.; Rossa, C. (2018). El aprendizaje servicio como pedagogia inclusiva en estudiantes con necesidades educativas especiales. RIDAS, Revista Iberoamericana de Aprendizaje Servicio, 5, 24-36. DOI10.1344/RIDAS2018.5.3 
humor; trastorno depresivo; trastorno distímico; trastornos neurológicos; parálisis cerebral infantil; epilepsia; trastornos de personalidad; psicosis; trastornos de apego. Muy a menudo, la esfera de las relaciones también produce dificultades inherentes a la esfera psicoafectiva (niños aislados, excesivamente dependientes, pasivos, etc.). Entre los trastornos específicos del aprendizaje encontramos los más tradicionales, como dislexia, disgrafía, discalculia, trastorno por déficit de atención con o sin hiperactividad, trastornos en la comprensión del texto, dificultades visoespaciales, dificultades habilidades motrices, torpeza, dispraxia del desarrollo, etc. Los estudiantes con dificultades de lenguaje o trastornos específicos del habla y la fonación también tienen dificultades para aprender y desarrollarse.

El mundo escolar está cada vez más atento a esas dificultades blandas, que se manifiestan a través de problemas motivacionales, perturbaciones de la autoimagen y la identidad, falta de autoestima, inseguridad y desorientación del proyecto de vida.

\section{Metodología}

En este trabajo hemos aplicado un método de educación comparada de tipo descriptivo-experimental o interpretativo fundado en la relevación sistemática de datos que conciernen proyectos de aprendizaje servicio en los cuales están involucrados estudiantes con NEE de cuatro países: Argentina, Suiza, España y Estados Unidos. El objetivo es evaluar y verificar la eficacia de la intervención de esta propuesta pedagógica innovadora en el campo de la inclusión educativa y social y delimitar los ámbitos de actuación, tratando de demostrar que los estudiantes con NEE, mas allá de la edad, patología y contexto cultural, son capaces de realizar acciones de participación activa en el territorio como agentes de transformación social.

El trabajo, desarrollado en etapas, comenzó con la comparación de diversos constructos teóricos sobre la definición de inclusión y buena didáctica inclusiva, como acaba de explicarse.

En un segundo momento se emprendió una fase de investigación evaluadora orientada a medir, a través de criterios de calidad, proyectos exitosos de aprendizaje servicio en los cuales participaron estudiantes con NEE. En esta fase, después de haber relevado la muestra, se elaboró una rúbrica de evaluación llegando a la construcción de cuatro modelos de inclusión mediante el aprendizaje servicio. Los instrumentos utilizados para la detección de datos fueron la narrativa de proyectos por parte de los protagonistas y entrevistas a los docentes responsables de proyectos de aprendizaje servicio. La muestra es numéricamente circunscripta a la cantidad de experiencias de aprendizaje servicio ya existentes.

El tercer momento consistió en la comparación entre diversos modelos de inclusión, área geográfica y tipologías de acción, a través del instrumento Cubo de comparación a multiniveles (Bray, Adamson y Mason, 2009).

Fiorin, I.; Rossa, C. (2018). El aprendizaje servicio como pedagogia inclusiva en estudiantes con necesidades educativas especiales. RIDAS, Revista Iberoamericana de Aprendizaje Servicio, 5, 24-36. DOI10.1344/RIDAS2018.5.3 


\section{Resultados y discusión}

El análisis demuestra que la verdadera inclusión es el resultado de un pacto social, de un compromiso común entre escuela y territorio. Una escuela inclusiva promueve la participación de los estudiantes fuera del aula, poniéndolos al servicio del bien común.

El estudio demuestra que el aprendizaje servicio es inclusivo porque didácticamente promueve el interés, la significatividad del aprendizaje, la motivación y la capacidad de proponer actividades desafiantes para todos los estudiantes (Booth y Ainscow, 2002; Gimeno Sacristan, 1999); una didáctica de laboratorio en problemas reales, se planifica por proyectos y se trabaja fuera del aula (Ianes, 2006) ; alianzas extraescolasticas; la diversidad por su flexibilidad, variedad y personalización en la organización de los tiempos, materiales y métodos (García Pastor y García Gómez, 2009); espacios de reflexión; cooperación y colaboración humana (Canevaro, 2006); la evaluación formativa; la participación y la autonomía (Trilla y Novella, 2001).

El principal aporte que emerge como resultado de este trabajo es la construcción de cuatro modelos de implementación de aprendizaje servicio e inclusión, tal y como se describe posteriormente.

En todos los proyectos evaluados se observan comportamientos prosociales (Roche, 1999) realizados por niños y adolescentes con NEE. A través de diversas acciones (ayuda, servicio, compartir, escucha, cuidado, solidaridad) se ofrece un servicio adecuado y deseado por los beneficiarios generando habitualmente una reciprocidad positiva. La orientación hacia las necesidades de los otros y el consecuente descentramiento de si mismos, particularmente limitado en las personas con algún tipo de psicopatología, se ven estimulados. Se observa reducción de la violencia y de la agresividad. Parece ser que el poseer un repertorio de comportamientos alternativos positivos haga crecer la capacidad prosocial y disminuir la violencia. En el aula y en la comunidad la frecuencia de comportamientos prosociales podría provocar un efecto multiplicador y por lo tanto de transformación social. Casi todos los proyectos manifiestan una mejora en la imagen de si mismos. Esto sucede por la percepción de éxito, de eficacia gracias a la constatación de beneficios hacia los demás. Los proyectos modifican sensiblemente también los factores ambientales. Se verifican remociones de obstáculos y barreras de todo tipo, arquitectónicas, psicológicas, políticas, educativas, comunicacionales que estaban presentes en el contexto social.

\subsection{Discusión}

La mayor parte de los proyectos analizados realizan servicios de atención directa a necesidades sociales (Ierullo y Ruffini, 2015) y esto no tanto por la demanda de los beneficiarios sino sobre todo por el tipo de actividad que pueden desarrollar los estudiantes con NEE. Generalmente el servicio que ofrecen es el resultado de lo que aprenden en los talleres (jardinería, carpintería, construccion de juegos, etc.). Se trata también de satisfacer

Fiorin, I.; Rossa, C. (2018). El aprendizaje servicio como pedagogia inclusiva en estudiantes con necesidades educativas especiales. RIDAS, Revista Iberoamericana de Aprendizaje Servicio, 5, 24-36. DOI10.1344/RIDAS2018.5.3 
necesidades sociales relacionales (compañía para los ancianos, actividades recreativas con otros chicos, etc.). Las otras categorías de servicio (transferencia de competencias, sensibilización, motorización del desarrollo) implican un nivel cognitivo superior y por eso son menos utilizadas.

La capacidad para involucrarse en otros tipos de servicios no debe ser subestimada. Aunque los casos sean raros, es importante analizar los proyectos en los cuales los estudiantes con NEE logran poner en acto otras competencias psicológicas. Esto depende de la condición física pero sobretodo de la condición ambiental y relacional, sobre todo de la confianza de los docentes y de la comunidad en el dar responsabilidad a las personas con NEE.

Ninguno de los proyectos evaluados reportan el uso de hardware y software para el aprendizaje y la comunicación y por lo tanto no preveen el uso de tecnologías de tipo compensativas. Se abre un espacio para la discusión respecto a la causa de la ausencia, y cual sería el resultado en términos de aprendizaje y de participación.

\section{Conclusiones}

El presente estudio demuestra que el aprendizaje servicio es una propuesta educativa inclusiva para todos los estudiantes, gracias a la cual también los estudiantes con NEE pueden alcanzar una plena inclusión como sujetos en la comunidad escolar y social, siendo protagonistas de acciones de mejora.
Los modos de implementar una práctica de aprendizaje servicio con estudiantes con NEE son muchas y se inspiran en cuatro modelos:

A. Deficit model: El proyecto tiene como destinatario personas con NEE. En este modelo entran un elevado numero de prácticas que van desde acciones de asistencia/beneficencia a aquellas que operan acciones de transformación para la remoción de un obstáculo sin involucrar personas con NEE en la realizaciones del proyecto. Las actitudes constituyen un continuum que van desde una perspectiva de compasión (como sentir dolor por el sufrimiento del otro) hacia un reconocimiento del valor del otro y de sus capacidades. Se constatan proyectos iniciados quizás con una intención de beneficencia, que, si son bien acompañados durante su desarrollo y planificación, con espacios de reflexión, permiten el desarrollo de relaciones de reciprocidad prosocial que ponen en primer lugar la dignidad del otro. Estas actividades favorecen el cambio de las actitudes y comportamientos estigmatizantes que se transforman en acogida y atribución de valor a la diversidad y permiten a los estudiantes ser ciudadanos responsables y comprometidos.

\section{B. Empowerment model: Los} estudiantes con NEE se transforman en agentes activos del cambio. Los chicos aprovechan sus puntos de fuerza y sus límites poniéndolos al servicio de los demás. Se posicionan en el centro de la atención de los docentes. Demuestran interés, iniciativa. Van con gusto a la escuela. Como verdaderos protagonistas experimentan un tipo de

Fiorin, I.; Rossa, C. (2018). El aprendizaje servicio como pedagogia inclusiva en estudiantes con necesidades educativas especiales. RIDAS, Revista Iberoamericana de Aprendizaje Servicio, 5, 24-36. DOI10.1344/RIDAS2018.5.3 
participación que permite no solo ser ejecutores sino que dan su opinión, hacen propuestas, evalúan. Cada estudiante considera como propio el proyecto y puede intervenir en él.

C. Reciprocal model: Se verifican acciones de inclusión recíproca, donde cada sujeto incluye al otro en términos de dar y recibir. El encuentro con el límite y la fragilidad de si mismos y del otro es visto como una oportunidad de relación. La orientación del proyecto es el compartir, resaltando relaciones de reciprocidad. El ambiente educativo es estimulante para todos y no deja atrás a ninguno. El proyecto nace como una actividad de escucha entre los estudiantes y los docentes, sea en forma individual (mentoring, consejo de clase, etc.) o colectiva (debates dentro del aula o consejos de clase, dinámicas de construcción cooperativas)

\section{Citizenship model: La ciudad} reconoce su rol educativo y asume en modo dinámico esta función, activando todo su potencia. Involucra los estudiantes con NEE en contextos sociales en los cuales ellos pueden dar su aporte o realizar un servicio significativo para los demás. Se construyen alianzas y redes en función de la inclusión de las personas más desventajazas de la ciudad. La comunidad reconoce la capacidad de participación de las personas que tiene algun tipo de discapacidad y propone actividades motivadoras, cercanas a sus intereses. El territorio se transforma en lugar de aprendizaje. Los entes locales se adaptan al ritmo de los estudiantes. Las reflexiones y las buenas practicas se transforman en un modelo compartido con la comunidad.

Los estudiantes con NEE pueden realizar diferentes tipos de servicios que van desde la asistencia directa a las necesidades sociales a otros más complejos, como acciones de sensibilización, transferencia de competencias y activación de procesos de desarrollo local.

El aprendizaje servicio es una propuesta pedagógica que contiene en si los criterios de una buena didáctica inclusiva. Dentro de ella conviven diferentes estrategias didácticas de tipo colaborativas como la tutoría, la mentoría y otras formas de relación entre estudiantes y docentes; el aprendizaje cooperativo y la educación ciudadana.

El aprendizaje servicio es una buena propuesta inclusiva porque promueve el encuentro con la comunidad, en función del desarrollo de las personas con NEE y el desarrollo del territorio. Se verifican cambios significativos en los factores ambientales, remoción de obstáculos o barreras de todo tipo, (arquitectónicas, psicológicas, políticas, educativas, comunicacionales), también disminuyen los comportamientos violentos y agresivos. En suma, la comunidad cambia su actitud, la percepción y comportamientos estigmatizantes.

\section{Referencias bibliográficas}

Adamson, B. y Morris, P. (2009). Comparare i curricula. En M. Bray; B. Adamson; M. Mason. Educazione comparata. Approcci e metodi di ricerca. Milano: FrancoAngeli.

Fiorin, I.; Rossa, C. (2018). El aprendizaje servicio como pedagogia inclusiva en estudiantes con necesidades educativas especiales. RIDAS, Revista Iberoamericana de Aprendizaje Servicio, 5, 24-36. DOI10.1344/RIDAS2018.5.3 
Ainscow, M. (2007). Towards a more inclusive education system. Where next for special schools? En R. Cigman. Included or Excluded? The challenge of the mainstream for some SEN children. Londres: Routledge.

Blanco, R. (2007). La equidad y la inclusión social: uno de los desafíos y de la escuela de hoy. Revista Latinoamericana de educación inclusiva. 1(1).

Booth, T. y Ainscow. M. (2002). Index for inclusion. Developing learning and participation in schools. Manchester: CSIE.

Bray, M.; Adamson, B. y Mason, M. (2009) Educazione comparata. Approcci e metodi di ricerca. Milano:

FrancoAngeli.

Canevaro A. (2006). Le logiche del confine e del sentiero. Trento:Erickson.

Dovigo, F.; Ianes, D. (edizione italiana a cura di) (2008) L'index per

l'inclusione. Promuovere l'apprendimento e la partecipazione nella scuola. Trento: Erickson.

Fiorin, I. (2016). (a cura di) Oltre I'aula. La proposta pedagogica del Service Learning. Milano: Mondadori.

Garcia Pastor, C. (2003). Segregación, Integración e Inclusión. Bordón. 55(1).

Garcia Pastor, C.; Garcia Gomez, M.S. (2009). Atención a la Diversidad: un Reto para la Convergencia Europea. Cuenca: Universidad de Castilla la Mancha.

Gimeno Sacristan, J. (1999). La construcción del discurso acerca de la diversidad y sus prácticas. Aula de innovación educativa, 8.

Ianes, D. (2006). La speciale normalità. Strategie d'integrazione e inclusione per le disabilità e i Bisogni Educativi Speciali. Trento: Erikson.

Milan, G. (2000). Educare all'Incontro. La pedagogia di Martin Buber. Roma: Città Nuova.

Roche, R. (1995). Psicología y educación para la prosocialidad. Barcelona: Universidad Autónoma de Barcelona.

Trilla, J. y Novella, A. (2001). Educación y participación social de la infancia. Revista Iberoamericana de Educación, 26.

UNESCO (1993) Declaración de Salamanca. Marco de acción para las necesidades educativas especiales. Paris: UNESCO.

Fiorin, I.; Rossa, C. (2018). El aprendizaje servicio como pedagogia inclusiva en estudiantes con necesidades educativas especiales. RIDAS, Revista Iberoamericana de Aprendizaje Servicio, 5, 24-36. DOI10.1344/RIDAS2018.5.3 